\title{
Unusual tumors associated with the hereditary nonpolyposis colorectal cancer syndrome
}

\author{
Russell R Broaddus ${ }^{1}$, Patrick M Lynch ${ }^{2}$, Karen H Lu ${ }^{3}$, Raja Luthra ${ }^{1}$ and Sara J Michelson ${ }^{4}$ \\ ${ }^{1}$ Department of Pathology; ${ }^{2}$ Department of GI Medicine and Nutrition; ${ }^{3}$ Department of Gynecologic Oncology \\ and ${ }^{4}$ Department of Clinical Cancer Genetics, University of Texas MD Anderson Cancer Center, Houston, \\ $T X, U S A$
}

\begin{abstract}
The molecular pathogenesis of tumors outside the usual tumor spectrum for hereditary nonpolyposis colorectal cancer (HNPCC) is currently controversial. Specifically, it is not known whether these tumors are related to defects in DNA mismatch repair or arise independently of this defect in these patients. Here, we report two young patients, each with a known MSH2 mutation in the family, who developed rare tumors (adrenal cortical carcinoma and anaplastic carcinoma of the thyroid) that are not usually associated with HNPCC. Both of these patients were members of families that fulfilled modified Amsterdam (Amsterdam II) criteria for this familial cancer syndrome. Both the adrenal tumor and the thyroid tumor showed complete loss of immunohistochemical expression for MSH2 protein. Neither tumor was considered microsatellite instabilityhigh following microsatellite instability analysis using the established National Cancer Institute panel of five microsatellite markers. To our knowledge, MSH2 defects in these types of tumors have not been previously reported in patients with the HNPCC syndrome. Our results suggest that microsatellite instability analysis using the National Cancer Institute panel of five microsatellite markers may not detect microsatellite instability in tumors that fall outside the usual tumor spectrum of this syndrome. Therefore, when analyzing unusual tumors in patients with known or suspected HNPCC syndrome, we advocate the performance of immunohistochemistry for mismatch repair gene products in addition to microsatellite instability analysis.
\end{abstract}

Modern Pathology (2004) 17, 981-989, advance online publication, 14 May 2004; doi:10.1038/modpathol.3800150

Keywords: adrenal cortical carcinoma; HNPCC; microsatellite instability; MSH2; thyroid anaplastic carcinoma

Hereditary nonpolyposis colorectal cancer (HNPCC) is an autosomal dominant genetic disorder characterized by early onset colorectal carcinoma. Several extracolonic malignancies are also integral to this syndrome, such as carcinomas of the endometrium, ovary, renal pelvis, ureter, stomach, and small intestine. $^{1-5}$ There are a number of reports of common tumors, such as breast cancer $^{6,7}$ and prostate cancer, ${ }^{8-11}$ arising in HNPCC patients that fall outside this usual tumor profile. It is currently controversial whether these tumors are coincidental or should be considered part of the HNPCC tumor spectrum.

There are isolated case reports of rare tumors arising in patients with HNPCC. For example, Sijmons et $a l^{12}$ reported a malignant fibrous histiocytoma (MFH) arising in a patient with a germline

Correspondence: Dr RR Broaddus, MD, PhD, Department of Pathology, Box 85, University of Texas MD Anderson Cancer Center, 1515 Holcombe Blvd., Houston, TX 77030, USA.

E-mail: rbroaddus@mdanderson.org

Received 2 February 2004; revised 25 March 2004; accepted 26 March 2004; published online 14 May 2004
MSH2 mutation and a family history that met the clinical Amsterdam I criteria for HNPCC. The authors contended that for this individual, the germline MSH2 mutation and MFH were causally linked because the tumor showed loss of MSH2 protein by immunohistochemistry expression and instability in five microsatellite markers. Similarly, a male with a germline $M L H 1$ mutation and a positive Amsterdam family history developed infiltrating ductal carcinoma of the breast 30 years after earlyonset colorectal cancer. ${ }^{13}$ The breast tumor in this patient showed microsatellite instability with BAT26 and BAT-40. Furthermore, the wild-type MLH1 allele was lost in the breast tumor tissue compared to normal tissue. It was therefore argued that for this male patient, the breast cancer was indeed related to the underlying germline mutation of MLH1. Last, a woman with an $\mathrm{MSH} 2$ germline mutation, ovarian cancer, and three metachronous colon cancers was found to also have an adrenal cortical carcinoma. ${ }^{14}$ Her family history did not meet the Amsterdam criteria for HNPCC. Immunohistochemical and microsatellite instability analysis was performed on one of the colon cancers and the adrenal cortical 
carcinoma. The colon tumor showed loss of MSH2 protein immunohistochemical expression and was microsatellite instability-high. However, the adrenal tumor retained MSH2 protein expression by immunohistochemistry and did not show instability in the traditional five microsatellites examined. It was therefore concluded that the development of the adrenal tumor was coincidental and not related to the MSH2 germline defect.

Here, we report two patients with HNPCC who each developed different rare tumors, adrenal cortical carcinoma and anaplastic thyroid cancer, not usually considered in the HNPCC tumor spectrum. Our data provide evidence suggesting that these tumors were not coincidental, but likely developed in association with the underlying germline defect in DNA mismatch repair.

\section{Materials and methods}

\section{Genetic Testing}

Following patient interviews and formal genetic counseling, full sequence determination of MLH1 and $\mathrm{MSH} 2$ was carried out by Myriad Genetics Laboratories. Briefly, DNA was extracted and purified from leukocytes derived from a peripheral blood sample. Following amplification by PCR, the DNA was directly sequenced in forward and reverse directions using fluorescent dye-labeled sequencing primers. Chromatographic tracings of each amplicon were analyzed and compared to those for a consensus wild-type sequence for each gene.

\section{Immunohistochemistry, Microsatellite Instability, and MLH1 Promoter Methylation}

Using standard immunohistochemical techniques, formalin-fixed, paraffin-embedded sections of tumor were analyzed by light microscopy for the immunohistochemical expression of MLH1 protein (G16815, 1:30, Pharmingen, San Diego, CA, USA) and MSH2 protein (FE11, 1:100, Oncogene Science, Boston, MA, USA). The immunoreactive proteins were visualized using the EnVision horseradish peroxidase kit (Dako, Carpinteria, CA, USA) for hMLH1 and the LSAB2 horseradish peroxidase kit (Dako) for hMSH2. Adjacent normal tissue and surrounding tissue lymphocytes served as internal positive controls for each case. Nuclear staining of the tumor was scored as either present or absent compared to the corresponding internal control.

Microsatellite instability analysis was performed using formalin-fixed, paraffin-embedded sections of tumor and corresponding normal tissue. DNA was extracted from microdissected areas of tumor and normal tissue from sections on glass slides. Following DNA amplification using fluorescent labeled primers, a panel of five microsatellites recommended by the $\mathrm{NCI}^{15}$, BAT25, BAT26, D2S123,
D5S346, and D17S250, was analyzed for allelic shift using a multiplex fluorescence-based PCR assay. The amplified PCR products were analyzed on an ABI Genetic Analyzer (PE/Applied Biosystems) using the GeneScan Analysis software provided by the manufacturer. Tumors showing allelic shift at two or more markers were classified as microsatellite instability-high, while those with allelic shift at one marker were classified as microsatellite instability-low. Tumors with no allelic shift at any marker were classified as microsatellite stable.

For the MLH1 methylation assay, DNA was isolated from formalin-fixed, paraffin-embedded tissue sections that were microdissected to provide relatively pure tumor samples. Following treatment with bisulfite, the DNA was amplified by PCR using primers that were specific for either the methylated (M) or the unmethylated (U) versions of MLH1 (MLH1-M forward, 5'-gatagcgatttttaacgc-3' and MLH1-M reverse, $5^{\prime}$-tctataaattactaaatctcttcg-3'; MLH1-U forward, $5^{\prime}$-agagtggatagtgatttttaatgt- $3^{\prime}$ and MLH1-U reverse, $5^{\prime}$-actctataaattactaaatctcttca- $3^{\prime}$ ). PCR products were separated on $6 \%$ polyacrylamide gels and visualized after staining with ethidium bromide. DNA extracted from the RKO colon carcinoma cell line was used as a positive control.

\section{Clinical reports}

\section{Patient 1}

Patient 1 , at age 34 years, developed generalized fatigue associated with hypertension and hypokalemia. A CT scan demonstrated a $4 \mathrm{~cm}$ left adrenal mass that was surgically removed and determined pathologically to be an adrenal cortical carcinoma (Figure 1a). After 3 years, he developed recurrence in the abdomen, which required surgical removal of the spleen, adjacent chest wall, portion of diaphragm, left kidney, and the tail of the pancreas. A year later, a mass was discovered in the cecum. Pathological evaluation of the right colectomy specimen revealed a moderately differentiated colonic adenocarcinoma invading the cecal muscularis propria. No lymph node metastasis was present. A liver biopsy performed at the time of colectomy, however, demonstrated metastatic adrenal cortical carcinoma. The patient died several months after colectomy. The family history (Figure 2a), which met the modified Amsterdam (Amsterdam II) criteria for HNPCC, was significant for colon cancer in the father (age 62 years) and endometrial cancer in two paternal aunts (ages 38 and 49 years). Following the death of Patient 1, his father underwent formal genetic counseling and testing and was found to have a suspected deleterious mutation of MSH2 (MSH2 variant IVS10 + 1G > A). Both of the paternal aunts who developed endometrial cancer at young ages underwent MSH2 single-site analysis and were found to carry the same MSH2 variant 


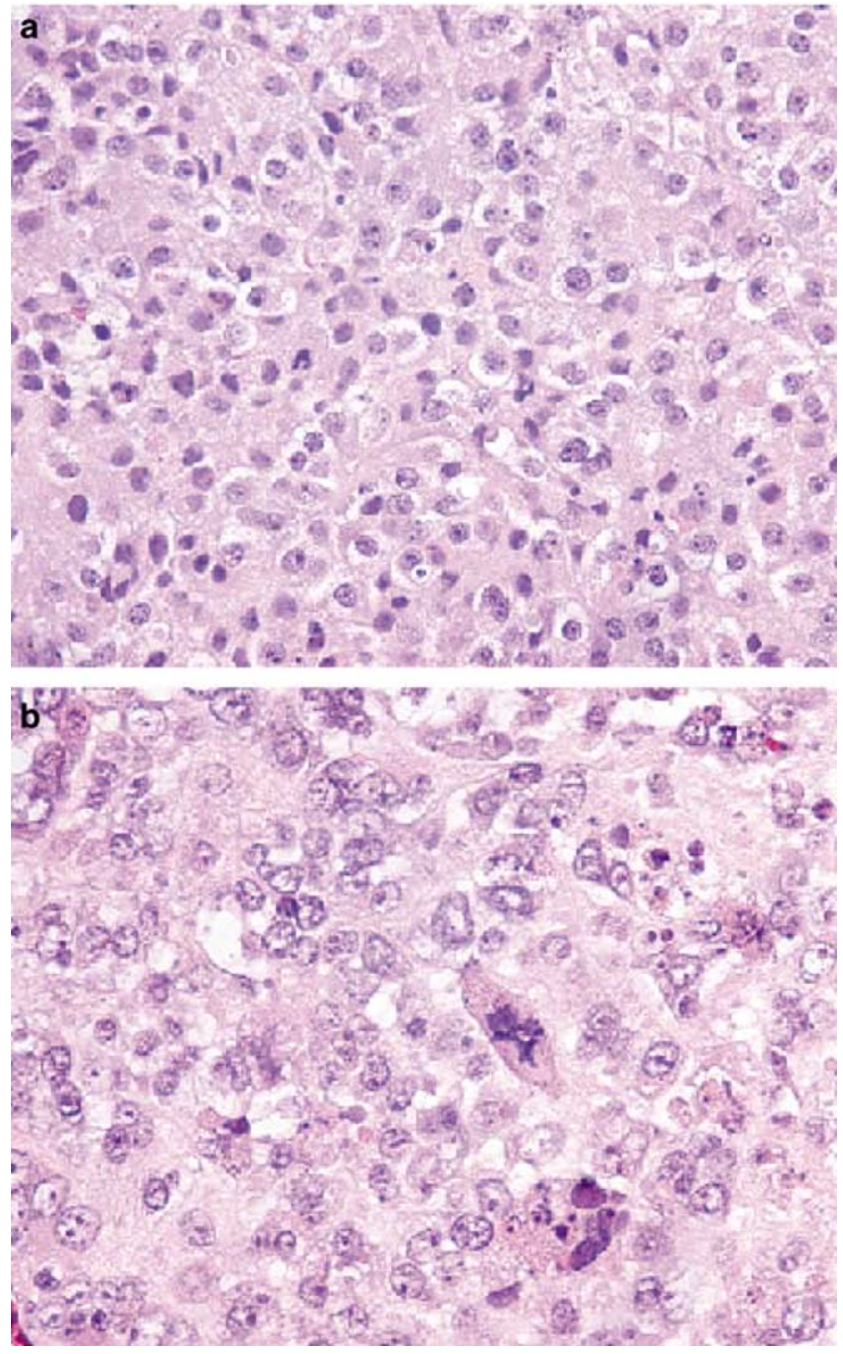

Figure 1 Adrenal cortical carcinoma from Patient 1 (a) and the thyroid anaplastic carcinoma from Patient 2 (b) (a and b, H\&E stain, $\times 200$ ). Both of these tumors had large areas in which there were very few contaminating normal cells. These were the areas that were specifically microdissected for DNA extraction and subsequent microsatellite instability analysis.

IVS10 $+1 \mathrm{G}>\mathrm{A}$, which supports the impression that this mutation is deleterious.

The colonic adenocarcinoma and the metastatic adrenal cortical carcinoma involving the liver were evaluated for the immunohistochemical expression of MLH1 and MSH2 proteins (Figure 3). Both the colon tumor and the adrenal tumor exhibited strong nuclear expression of MLH1. Both tumors showed loss of nuclear expression of MSH2, corresponding to the suspected gene defect in the family. Importantly, adjacent normal tissue in each of these sections demonstrated positive nuclear expression of MSH2.

Microsatellite instability analysis, using the panel of five microsatellites recommended by the NCI, ${ }^{15}$ was performed for both the colonic and adrenal carcinoma. All five microsatellites were unstable in
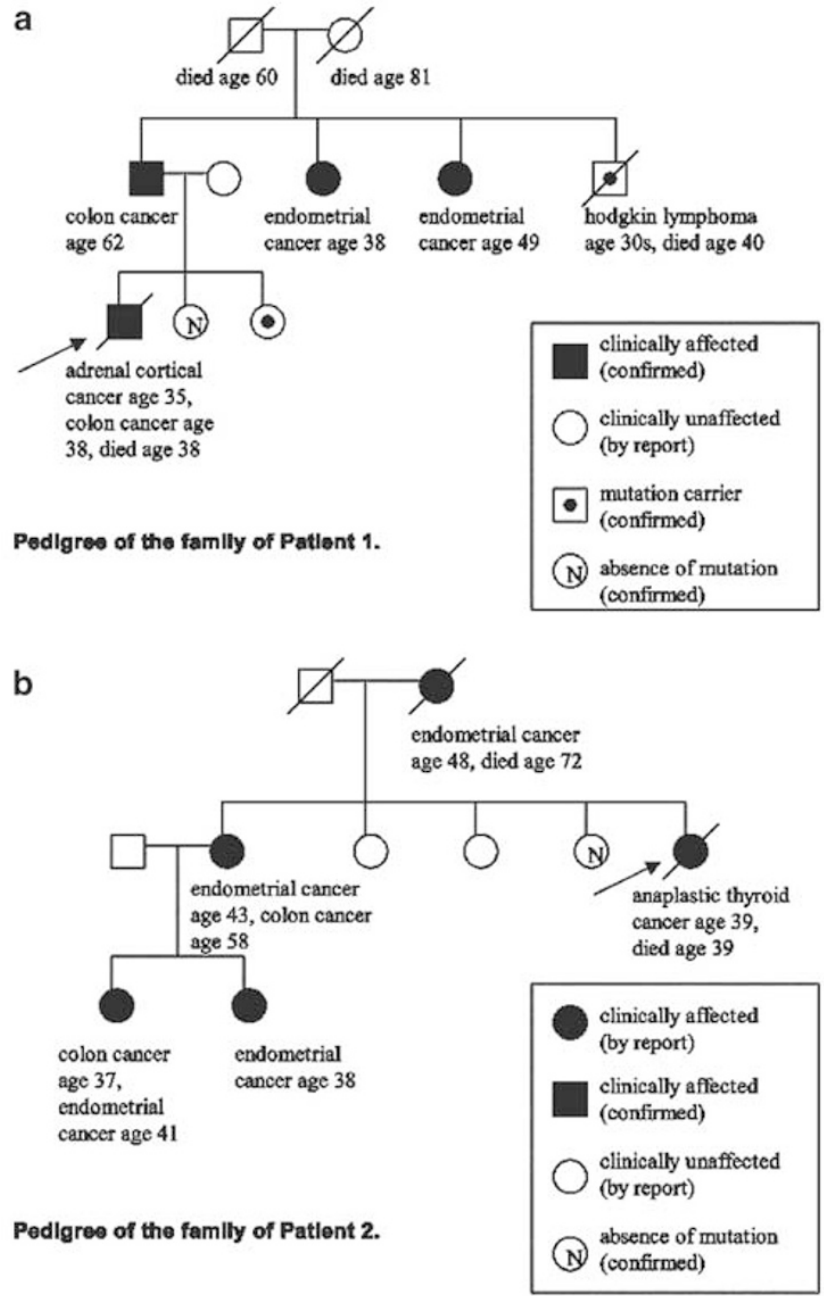

Figure 2 Pedigrees for Patient 1 (a) and Patient 2 (b).

the colon tumor (microsatellite instability-high; Figure 4). However, none of the microsatellites showed allelic shift in the adrenal carcinoma (microsatellite stable; Figure 4).

\section{Patient 2}

Patient 2, a 39-year-old woman, was previously in good health until she noted a right neck mass. Upon physical examination, this was determined to be a right thyroid nodule. Fine-needle aspiration revealed findings consistent with a colloid nodule vs a follicular neoplasm. Pathological evaluation of the surgical resection specimen, however, demonstrated an anaplastic carcinoma of the thyroid (Figure 1b). At the time of the thyroid surgery, there were two lung nodules and a possible pancreatic metastasis. At 4 months after starting adjuvant chemotherapy and radiation treatment, the residual tumor in the neck progressively grew until the patient developed stridor and dyspnea. The patient died approximately 7 months after the initial diagnosis. The 

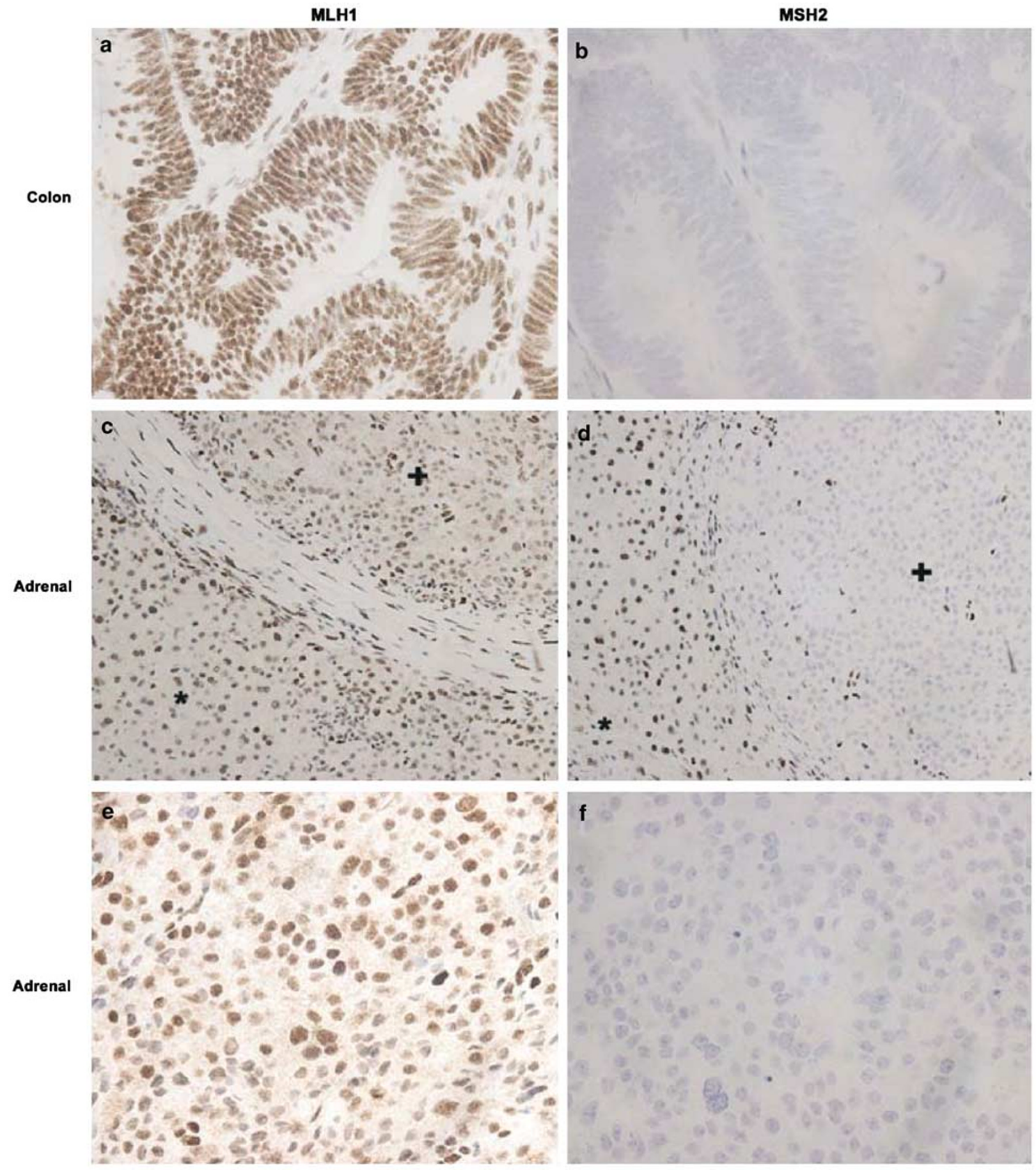

Figure 3 Immunohistochemical analysis of MLH1 and MSH2 protein expression in the colonic adenocarcinoma and the adrenal cortical carcinoma for Patient $1(\mathbf{a}, \mathbf{b}, \mathbf{e}$, and $\mathbf{f}, \times 200 ; \mathbf{c}$ and $\mathbf{d}, \times 100)$. The colonic adenocarcinoma retained expression of MLH1 (a), but lost expression of MSH2 (b), consistent with the underlying mutation in MSH2 detected in the father of Patient 1 . Both (c) and (d) demonstrate normal liver $(*)$ separated by a fibrous capsule from the metastatic adrenal carcinoma $(+)$. The normal liver and metastatic adrenal carcinoma expressed MLH1 ((c); higher magnification of adrenal carcinoma only in (e)). The normal liver, but not the adjacent metastatic adrenal carcinoma, expressed MSH2 ((d); higher magnification of adrenal carcinoma only in (f)). The few, scattered hMSH2positive cells within the tumor in (d) are lymphocytes and fibroblasts.

family history (Figure 2b) was significant for endometrial cancer in the mother (age 48 years) and one niece (age 38 years) and for endometrial cancer plus colon cancer in a sister (endometrial cancer, age 43 years; colon cancer, age 58 years) and another niece (colon cancer, age 37 years; endometrial cancer age 41 years). As for Patient 1, the family history for Patient 2 also met the modified Amsterdam (Amsterdam II) criteria for HNPCC. The niece with both colon cancer and endometrial 
a
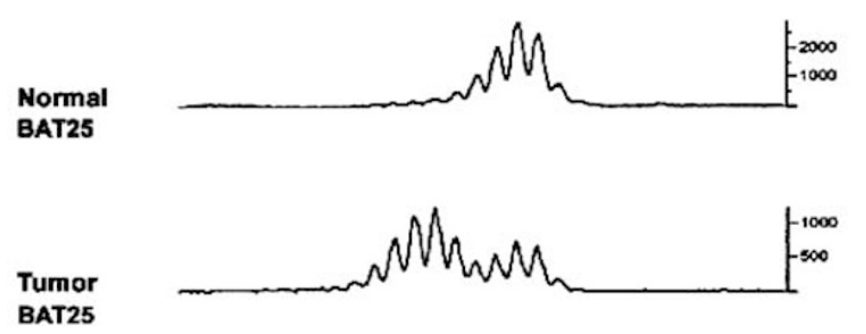

b

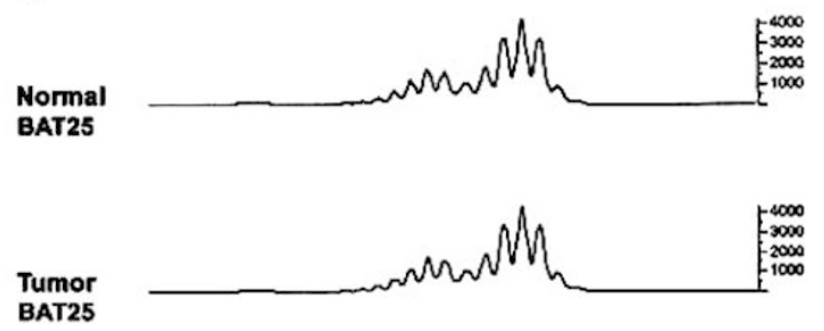

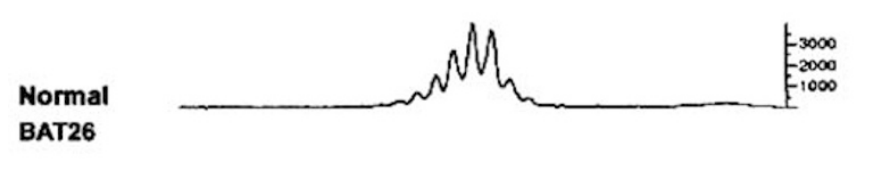
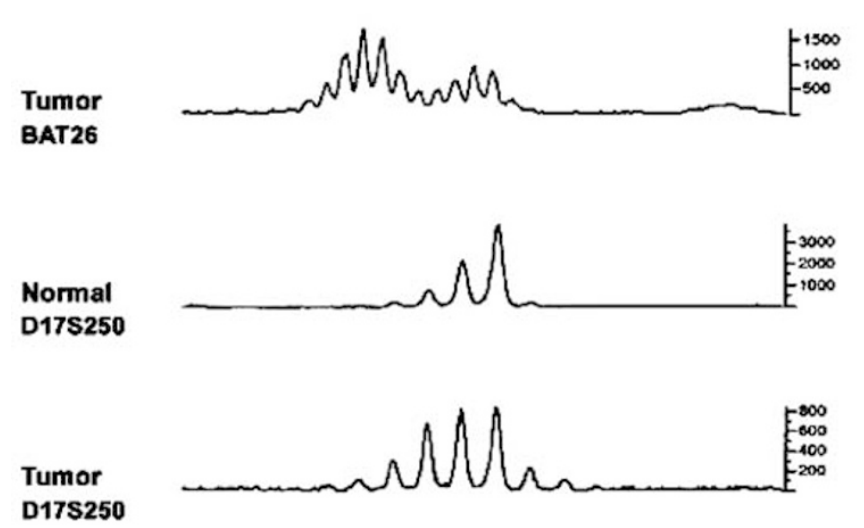

Normal BAT26

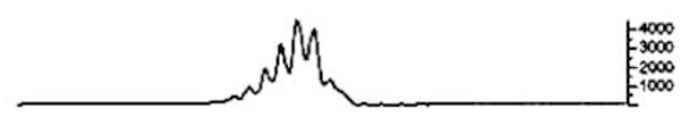

Tumor

BAT26

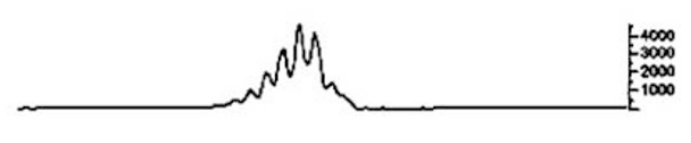

Normal

D17S250

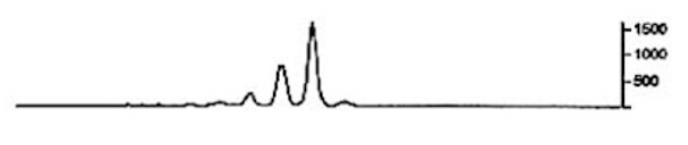

Tumor

D175250

Figure 4 Representative results of microsatellite instability analysis for the colonic adenocarcinoma (a) and adrenal cortical carcinoma (b) for Patient 1. The colon tumor had allelic shift (exhibited in the analysis as additional, abnormal peaks in tumor DNA compared to normal DNA) in all five microsatellites examined. However, no allelic shift was noted in the microsatellites for the adrenal tumor (results for D2S123 and D5S346 not shown).

cancer underwent $M L H 1$ and MSH2 full sequence analysis and was found to have a deleterious mutation of MSH2 (mutation Q824X, resulting in premature truncation of the $\mathrm{MSH} 2$ protein at amino acid 824).

Immunohistochemical expression of MLH1 and MSH2 proteins was examined in the thyroid tumor from Patient 2. For comparison, the endometrial tumor from the niece who underwent formal genetic testing was also examined; the colon tumor from this patient was not available. In addition, we examined the colon carcinoma from the sister of Patient 2. The endometrial carcinoma, colon adenocarcinoma, and the thyroid carcinoma each had strong nuclear expression of MLH1, but lacked nuclear expression of MSH2 (Figure 5). These immunohistochemistry results correlate with the results of genetic testing for the niece, which revealed a deleterious mutation of $\mathrm{MSH} 2$. Microsatellite instability analysis was performed on the thyroid tumor and the colon tumor only, as there was insufficient endometrial tumor for adequate DNA extraction. The colon cancer showed allelic shift in three of the five microsatellites, BAT25, BAT26, and D17S250 (microsatellite instability- high; Figure 6). For the thyroid tumor, only one of the five microsatellites, D17S250, demonstrated allelic shift (Figure 6), so this tumor was classified as microsatellite instability-low.

\section{Methylation of $M L H 1$}

In sporadic tumors, especially sporadic colorectal adenocarcinoma, microsatellite instability may be secondary to methylation of $M L H 1$, with subsequent loss of MLH1 protein expression. ${ }^{16,17}$ Therefore, MLH1 methylation for both the adrenal tumor (Patient 1) and the thyroid tumor (Patient 2) was analyzed by methylation-specific PCR (Figure 7). Neither the adrenal tumor nor the thyroid tumor demonstrated methylation of MLH1. These results support the positive immunohistochemical expression of MLH1 protein observed in both of these neoplasms (Figures 3 and 5). Furthermore, lack of MLH1 methylation supports the assertion that the adrenal and thyroid tumors were associated with the loss of MSH2 protein observed by immunohistochemistry (Figures 3 and 5) and the MSH2 mutations detected in each family. 


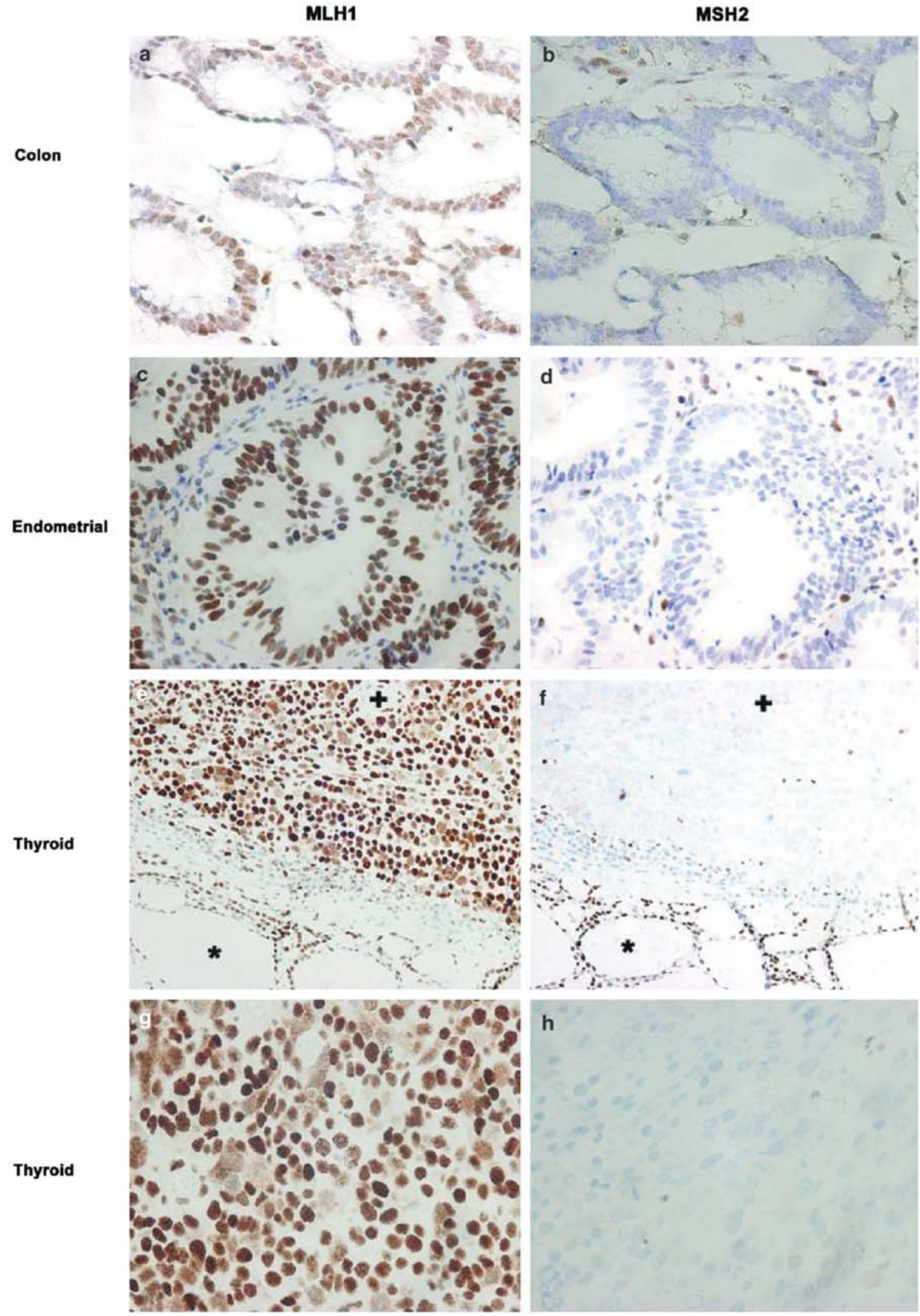


a

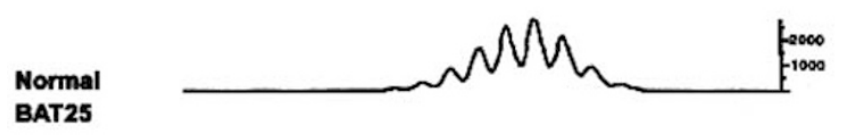

b

Normal BAT25
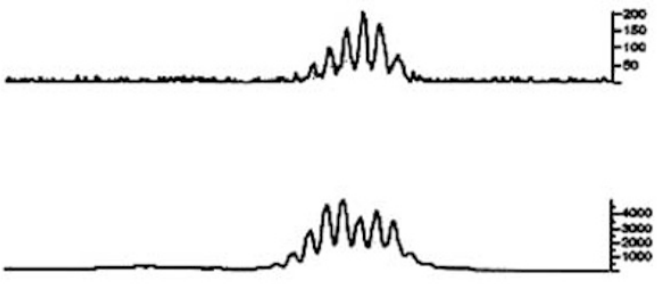

Tumor

BAT25

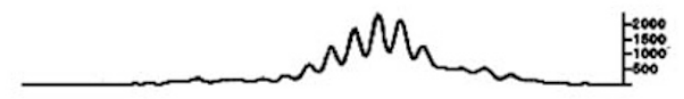

Normal

BAT26

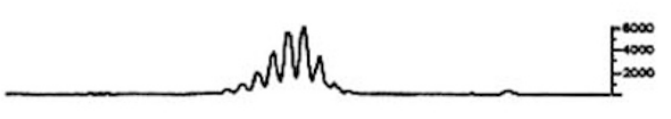

BAT26

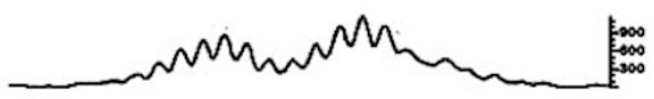

Tumor

BAT26

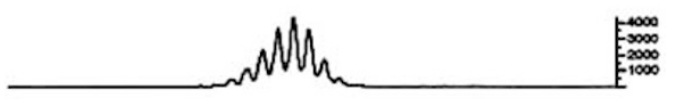

BAT26

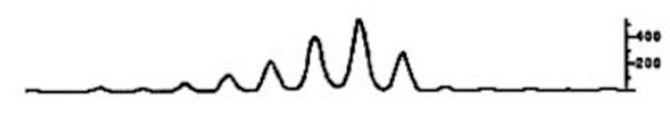

Normal

D175250

Normal D178250
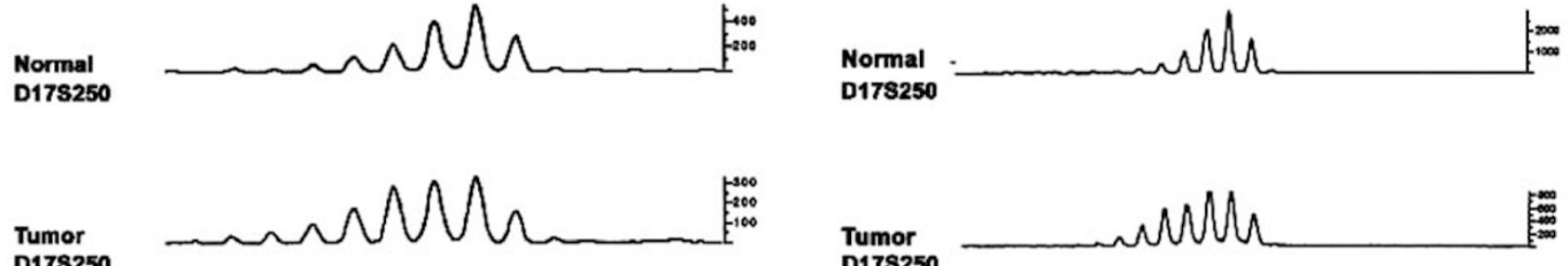

Tumor

D17\$250

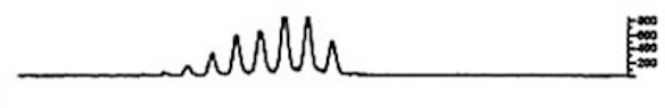

Figure 6 Representative results of microsatellite instability analysis for the thyroid tumor (b) from Patient 2 and the colon cancer (a) from an affected sister. The colon tumor exhibited allelic shift (additional, abnormal peaks in tumor DNA compared to normal) in all five microsatellites examined and was thus classified as microsatellite instability-high. The thyroid tumor only had allelic shift in one microsatellite, D17S250, and was thus classified as microsatellite instability-low (results not shown for D2S123 and D5S346).

\section{Discussion}

The etiology of tumors in HNPCC patients that fall outside the usual HNPCC tumor spectrum is controversial. For cancers that are common in the general population, such as breast cancer and prostate cancer, it is difficult to prove that these tumors are related to the molecular defect in HNPCC. However, when rare tumors arise in HNPCC patients, it is more enticing to think that these tumors were secondary to the defect in DNA mismatch repair. In this paper, we have summarized the tumor findings from two patients, each from a family with a documented MSH2 mutation. Both families fulfilled modified Amsterdam criteria for HNPCC. Both of these patients had rare tumors, adrenal cortical carcinoma (Patient 1) and anaplastic carcinoma of the thyroid (Patient 2) that are not traditionally associated with HNPCC. Since both of these tumors are rare in the general population, we felt that the association with HNPCC was more than coincidental. We have provided evidence that is highly suggestive that these tumors, though unusual, were associated with the underlying defect in MSH2. In support of this assertion, both the adrenal tumor and the thyroid tumor demonstrated loss of MSH2 protein expression by immunohistochemistry, but retained expression of MLH1 (Figures 3 and 5). This staining pattern was similar to that seen in the more common HNPCC-related malignancies, colon cancer and endometrial cancer, examined for each family (Figures 3 and 5). Also, adrenal cortical carcinoma and anaplastic carcinoma of the thyroid more commonly affect older patients. Patient 1 , with

Figure 5 Immunohistochemical analysis of MLH1 and MSH2 protein expression for Patient 2 (a-d and $\mathbf{g}-\mathbf{h}, \times 200$; e and $\mathbf{f}, \times 100$ ). Results for the patient's thyroid anaplastic carcinoma $(\mathbf{e}-\mathbf{h})$ were compared to those for a colon adenocarcinoma (a and $\mathbf{b})$ from an affected sister and an endometrial endometrioid adenocarcinoma (c and d) from an affected niece. The colon cancer and the endometrial cancer both expressed MLH1, but did not express MSH2, consistent with the underlying MSH2 mutation detected in the niece. Both (e) and (f) demonstrate normal thyroid (*) with adjacent anaplastic carcinoma ( +). The normal thyroid and adjacent carcinoma expressed MLH1 ((e); higher magnification of thyroid carcinoma only in (g)). In contrast, the thyroid tumor did not express MSH2, while the adjacent normal thyroid gland retained expression ((f); higher magnification of tumor only in (h)). 


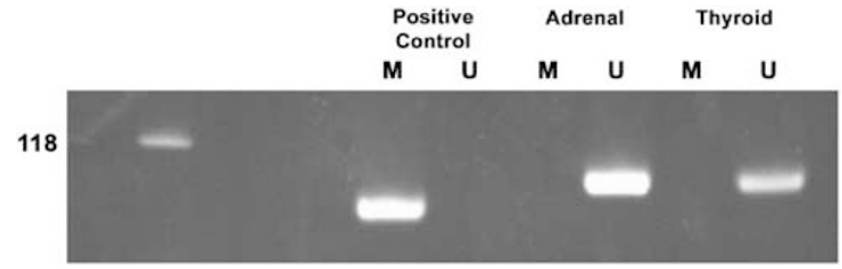

Figure 7 Analysis of $M L H 1$ methylation by methylation-specific PCR. Methylation of MLH1 is present in the positive control (RKO colon carcinoma cell line), but not in the adrenal tumor or thyroid tumor. These results are complimentary to those presented in Figures 3 and 5, which showed that immunohistochemical expression of MLH1 protein is retained in these tumors. U, unmethylated; $\mathrm{M}$, methylated.

adrenal cortical carcinoma, was 34 years old when diagnosed with this tumor; the average age of diagnosis for this malignancy is approximately 48 years old. ${ }^{18,19}$ Patient 2, with anaplastic thyroid carcinoma, was diagnosed at age 39 years; the average age of diagnosis for this tumor is 66.5 years. ${ }^{20}$ The early age of onset for both of these patients is again highly suggestive that these tumors were related to the underlying $\mathrm{MSH} 2$ defect and not coincidental.

Although both the adrenal and thyroid carcinomas showed loss of MSH2 immunohistochemical expression, neither tumor was microsatellite instability-high (Figures $4 \mathrm{~b}$ and $6 \mathrm{~b}$ ). For comparison, the colon cancers analyzed from each family were microsatellite instability-high (Figures 4a and 6a). Similar disparate patterns in microsatellite instability were also recently noted in colon cancers $(n=44)$ vs endometrial cancers $(n=57)$ from HNPCC patients with germline mutations in either MLH1 or MSH $2 .^{21}$ In this study, $23 \%$ of the endometrial cancers studied showed no evidence of microsatellite instability in any of the extended panel of 12 markers examined. Overall, the mean number of unstable markers per tumor was significantly lower in the endometrial cancers than the colon cancers. ${ }^{21}$

The reasons for decreased or lack of microsatellite instability in extracolonic cancers in HNPCC are not clear. One possible explanation is contaminating normal cells admixed with the tumor cells when extracting DNA for microsatellite analysis. As seen in Figure 1, however, we were able to microdissect areas from both the adrenal carcinoma and the thyroid anaplastic carcinoma that were greater than $90 \%$ tumor cells, with very few contaminating normal cells. Also, from our more extensive experience with colon cancers from HNPCC patients, there are far more contaminating inflammatory cells and stromal cells in colon cancers, yet these tumors are consistently microsatellite instability-high. Another possible explanation is that there may be intratumoral heterogeneity such that a tumor is composed of microsatellite instability-high and microsatellite instability-stable tumor cell populations. ${ }^{22}$ Again, for the adrenal and thyroid tumors, we feel that this is unlikely, as immunohistochemistry demonstrated only rare MSH2-positive stromal cells for each of these cases (Figures 3 and 5). A third possible explanation for the decreased microsatellite instability in extra-colonic cancers is that cis and/or trans acting tissue-specific factors, along with defects in MLH1 or MSH2, play a role in determining instability of microsatellites. ${ }^{23}$ Evidence that supports this explanation comes from transgenic mouse models of unstable CAG CTG repeats, which are expanded in diseases such as myotonic dystrophy type 1, Huntington's disease, fragile $\mathrm{X}$ syndrome, Friedreich's ataxia, and spinocerebellar ataxias. ${ }^{24}$ Cell cultures derived from the eyes, kidneys, and lungs from these transgenic mice demonstrated different levels of repeat instability, independent of cell proliferation rate. ${ }^{23}$ Similar observations have been made in humans with myotonic dystrophy type 1, as larger repeat lengths are consistently observed in muscle compared to blood DNA. ${ }^{25-27}$ Although we favor the possibility of tissue-specific factors influencing microsatellite instability, there is currently no experimental evidence to support this in HNPCC.

A final, unresolved issue pertains to the risk of relatives of Patient 1 and Patient 2 developing adrenal cortical carcinoma or anaplastic thyroid cancer, respectively. This is not a trivial concern, as both of these malignancies were fatal. It is known that some HNPCC families are prone to developing certain malignancies. For example, families with an MSH6 mutation have been found to have a higher number of endometrial cancer, ${ }^{28-31}$ distal colon cancer, ${ }^{28}$ and transitional cell carcinoma of the urinary tract. ${ }^{30}$ However, we are not aware of any reports in the literature of HNPCC families with larger numbers of rare tumors. Therefore, the risk of developing these rare neoplasms in HNPCC remains indeterminate. Since these tumors are rare in the general population, we must assume at this point that they are also rare in HNPCC families.

Based on the results from the two patients reported here and on the fact that adrenal cortical carcinoma and anaplastic thyroid carcinoma tend to arise in older individuals in the general population, younger patients who develop these tumors should be carefully screened for the possibility of HNPCC. Such screening could initially consist of the careful documentation of family history of cancer. If the family history meets Amsterdam or modified Amsterdam criteria, then the tumor tissue should be examined for the immunohistochemical expression of MLH1 and MSH2 and microsatellite instability. If these tests are informative, then formal genetic testing should be pursued.

\section{Acknowledgements}

We gratefully acknowledge the expertise of Mario Luna, MD, and Harry Evans, MD, in the pathological analysis of these tumors. 


\section{References}

1 Aarnio M, Mecklin JP, Altonen L, et al. Lifetime risk of different cancers in HNPCC syndrome. Int J Cancer 1995;64:430-433.

2 Dunlop MG, Farrington SM, Carothers AD, et al. Cancer risk associated with germline DNA mismatch repair gene mutations. Hum Mol Genet 1997;6: 105-110.

3 Lynch HT, Smyrk T. HNPCC (Lynch Syndrome): an updated review. Cancer 1996;78:1149-1167.

4 Mecklin JP, Jarvinen H. Tumor spectrum in cancer family syndrome (HNPCC). Cancer 1991;68:1109-1112.

5 Watson P, Lynch HT. Extracolonic cancer in HNPCC. Cancer 1993;71:677-685.

6 Muller A, Edmonston TB, Corao DA, et al. Exclusion of breast cancer as an integral tumor of hereditary nonpolyposis colorectal cancer. Cancer Res 2002; 62:1014-1019.

7 Risinger JI, Barrett JC, Watson P, et al. Molecular genetic evidence of the occurrence breast cancer as an integral tumor in patients with the hereditary nonpolyposis colorectal carcinoma syndrome. Cancer (Phila) 1996;77:1836-1843.

8 Bapat BV, Madlensky L, Temple LKF, et al. Family history characteristics, tumor microsatellite instability, and germline MSH2 and MLH1 mutations in hereditary colorectal cancer. Hum Genet 1999;104: 167-176.

9 Honchel R, Halling KC, Schaid DJ, et al. Microsatellite instability in Muir-Torre syndrome. Cancer Res 1994;97:159-163.

10 Scott RJ, McPhillips M, Meldrum CJ, et al. Hereditary nonpolyposis colorectal cancer in 95 families: differences and similarities between mutation-positive and mutation-negative kindreds. Am J Hum Genet 2001; 68:118-127.

11 Soravia C, van der Klift $\mathrm{H}$, Brundler M-A, et al. Prostate cancer is part of the hereditary non-polyposis colorectal cancer (HNPCC) tumor spectrum. Am J Med Genet 2003;121A:159-162.

12 Sijmons R, Hofstra R, Hollema $\mathrm{H}$, et al. Inclusion of malignant fibrous histiocytoma in the tumour spectrum associated with hereditary non-polyposis colorectal cancer. Genes Chromosomes Cancer 2000; 29:353-355.

13 Boyd J, Rhei E, Federici MG, et al. Male breast cancer in the hereditary nonpolyposis colorectal cancer syndrome. Breast Cancer Res Treat 1999;53:87-91.

14 Berends MJW, Cats A, Hollema H, et al. Adrenocortical adenocarcinoma in an $\mathrm{MSH} 2$ carrier: coincidence or causal relation? Hum Pathol 2000;31:1522-1527.

15 Boland CR, Thibodeau SN, Hamilton SR, et al. A National Cancer Institute workshop on microsatellite instability for cancer detection and familial predisposition: development of international criteria for the determination of microsatellite instability in colorectal cancer. Cancer Res 1998;58:5248-5257.

16 Kane MF, Loda M, Gaida GM, et al. Methylation of the $h M L H 1$ promoter correlates with lack of expression of
hMLH1 in sporadic colon tumors and mismatch repairdefective human tumor cell lines. Cancer Res 1997;57:808-811.

17 Herman JG, Umar A, Polyak K, et al. Incidence and functional consequences of $h M L H 1$ promoter hypermethylation in colorectal carcinoma. Proc Natl Acad Sci USA 1998;95:6870-6875.

18 Icard P, Chapuis Y, Andreassian B, et al. Adrenocortical carcinoma in surgically treated patients: a retrospective study on 156 cases by the French Association of Endocrine Surgery. Surgery 1992;112:972-980.

19 Nader S, Hickey RC, Sellin RV, et al. Adrenal cortical carcinoma: a study of 77 cases. Cancer 1983;52: 707-711.

20 Carcangiu ML, Steeper T, Zampi G, et al. Anaplastic thyroid cancer: a study of 70 cases. Am J Clin Pathol 1985;83:135-158.

21 Kuismanen SA, Moiso AL, Schweizer P, et al. Endometrial and colorectal tumors from patients with hereditary nonpolyposis colon cancer display different patterns of microsatellite instability. Am J Pathol 2002;160:1953-1958.

22 Barnetson R, Jass J, Tse R, et al. Mutations associated with microsatellite unstable colorectal carcinomas exhibit widespread intratumoral heterogeneity. Genes Chromosomes Cancer 2000;29:130-136.

23 Gomes-Pereira M, Fortune MT, Monckton DG. Mouse tissue culture models of unstable triplet repeats: in vitro selection for larger alleles, mutational expansion bias and tissue specificity, but no association with cell division rates. Hum Mol Genet 2001;10:845-854.

24 Cummings CJ, Zoghbi HY. Fourteen and counting: unraveling trinucleotide repeat diseases. Hum Mol Genet 2000;9:909-916.

25 Anvret M, Ahlberg G, Grandell U, et al. Larger expansions of the CTG repeat in muscle compared to lymphocytes from patients with myotonic dystrophy. Hum Mol Genet 1993;2:1397-1400.

26 Ashizawa T, Dubel JR, Harati Y. Somatic instability of CTG repeat in myotonic dystrophy. Neurology 1993;43:2674-2678.

27 Thornton CA, Johnson KJ, Moxley RT. Myotonic dystrophy patients have larger CTG expansions in skeletal muscle than in leukocytes. Ann Neurol 1994;35:104-107.

28 Berends MJW, Wu Y, Sijmons RH, et al. Molecular and clinical characteristics of MSH6 variants: an analysis of 25 index carriers of a germline variant. Am J Hum Genet 2002;70:26-37.

29 Miyaki M, Konishi M, Tanaka K, et al. Germline mutation of MSH6 as the cause of hereditary nonpolyposis colorectal cancer. Nat Genet 1997;17: 271-272.

30 Wagner A, Hendricks Y, Meijers-Heijboer EJ, et al. Atypical HNPCC owing to MSH6 germline mutations: analysis of a large Dutch pedigree. J Med Genet 2001;38:318-322.

31 Wijnen J, de Leeuw W, Vasen $\mathrm{H}$, et al. Familial endometrial cancer in female carriers of MSH6 mutations. Nat Genet 1999;23:142-144. 